\title{
PO072
}

\section{CACULATION OF CCT AND DUV BASED ON POLYNOMIAL UP TO THIRD ORDER}

\author{
Cheng Gao et al.
}

DOI 10.25039/x46.2019.PO072

from

CIE x046:2019

\section{Proceedings}

of the

29th CIE SESSION

Washington D.C., USA, June 14 - 22, 2019

(DOI 10.25039/x46.2019)

The paper has been presented at the 29th CIE Session, Washington D.C., USA, June 14-22, 2019. It has not been peer-reviewed by CIE.

(c) CIE 2019

All rights reserved. Unless otherwise specified, no part of this publication may be reproduced or utilized in any form or by any means, electronic or mechanical, including photocopying and microfilm, without permission in writing from CIE Central Bureau at the address below. Any mention of organizations or products does not imply endorsement by the CIE.

This paper is made available open access for individual use. However, in all other cases all rights are reserved unless explicit permission is sought from and given by the $\mathrm{CIE}$.

CIE Central Bureau

Babenbergerstrasse 9

A-1010 Vienna

Austria

Tel.: +4317143187

e-mail: ciecb@cie.co.at

www.cie.co.at 


\title{
CACULATION OF CCT AND DUV BASED ON POLYNOMIAL UP TO THIRD ORDER
}

\author{
Gao, C. ${ }^{1}$, Wang, Z. ${ }^{1}, \mathrm{XU}, \mathrm{Y} .{ }^{1}, \mathrm{Li}, \mathrm{C} . \mathrm{J}^{1{ }^{*}}$ \\ ${ }^{1}$ University of Science and Technology Liaoning, Anshan, CHINA \\ cjliustl@sina.com
}

DOI 10.25039/x46.2019.PO072

\begin{abstract}
Recently, Ohno [LEUKOS, 10:47-55, 2014] proposed a method for computing the correlated colour temperature (CCT) of a light source, which is a combination of the triangle solution and the parabolic solution. In this paper, a hybrid method consisting of third order polynomial solution, the triangle and parabolic solutions is proposed to further enhance the Ohno method. Simulation results shows the proposed method performs better than the Ohno method does in predicting CCT and Duv, especially when the CCT is large.
\end{abstract}

Keywords: CCT, Duv, triangle solution, parabolic solution, third order polynomial solution.

\section{Introduction}

The spectral power distribution (SPD) of a Planckian radiator [1], denoted by $M(\lambda, T)$, can be completely determined from its absolute temperature $T$ in Kelvin $(K)$ using the expression:

$$
M(\lambda, T)=c_{1} \lambda^{-5}\left(e^{c_{2} /(\lambda T)}-1\right)^{-1},
$$

where constant $c_{1}=3.741832 \times 10^{-16} \mathrm{Wm}^{2}$ and $c_{2}=1.4388 \times 10^{-2} \mathrm{~m} \mathrm{~K}$. The colour temperature $T$ of a light source is the colour temperature of a Planckian radiator which emits radiation of the same chromaticity as the light source. When a light source has a chromaticity which is not the same as the one of the Planckian radiator, the term "correlated colour temperature" (CCT) is used, defined as the temperature of the Planckian radiator whose chromaticity is the one nearest to that of the light source in a uniform chromaticity scale (UCS) diagram [1]. Specifically, the UCS diagram recommended up to now by the International Commission on Illumination (CIE) for CCT computation is the CIE 1960 UCS diagram [1], where the chromaticity coordinates are given by:

$$
\begin{aligned}
& u=4 x /(-2 x+12 y+3), \\
& v=6 y /(-2 x+12 y+3)
\end{aligned}
$$

and $x, y$ are the chromaticity coordinates of the light source in the CIE 1931 coordinate system [2]. Note that the uniform chromaticity coordinates $(u, v)$ currently are not used anymore, instead, a better uniform spaces $\left(u^{\prime}, v^{\prime}\right)$ are recommended by CIE [3]. However, for the computation of CCT, chromaticity coordinates $(u, v)$ is still considered. Since $(u, v)=\left(u^{\prime}, \frac{2}{3} v^{\prime}\right)$, we can compute CCT in space $\left(u^{\prime}, v^{\prime}\right)$. However, for simplicity, in this paper the space $(u, v)$ is used.

Let $u, v$ be the chromaticity coordinates of the light source/illuminant, and $u(T), v(T)$ be the chromaticity coordinates of the Planckian radiator with colour temperature $T$. Then, the square of the distance between $(u, v)$ and $(u(T), v(T))$ is the function $f(T)$ :

$$
f(T)=[u-u(T)]^{2}+[v-v(T)]^{2},
$$

and the computation of the CCT is just a minimization of this objective function $f(T)$ with $T$. 
CCT, however, provides only one dimension of the chromaticity $(u, v)$ of the light source considered, and there is another dimension, which is the position of chromaticity with respect to Planckian locus, as noted by Ohno [4]. For this purpose, " $D_{u v}$ " as the distance from the Planckian locus have been used. Suppose for the given light source with chromaticity coordinates $(u, v)$, and the function $f(T)$ reaches its minimum when $T=T_{0}$, which means the CCT is $T_{0}$ for the given light source, and thus the $D_{u v}$ for the given light source is given by [4]:

$$
D_{u v}=s \sqrt{\left[u-u\left(T_{0}\right)\right]^{2}+\left[v-v\left(T_{0}\right)\right]^{2}}
$$

where, $s=1$ if $v-v\left(T_{0}\right) \geq 0$ and otherwise $s=-1$.

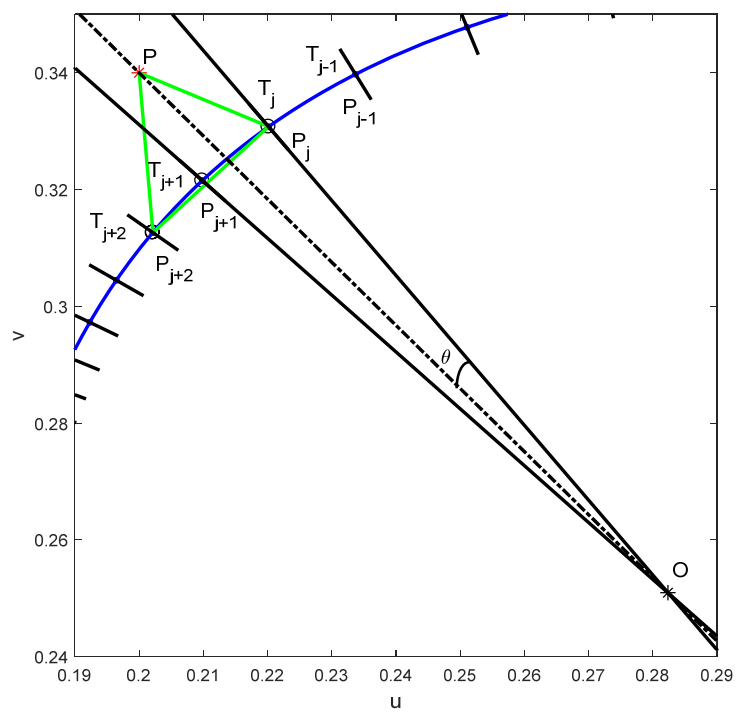

\section{Figure 1 - Illustration for Robertson and Ohno methods for finding correlated colour temperature}

For estimating the correlated colour temperature (CCT) of a light source, a nonlinear optimization problem must be solved as discussed above and the minimization problem has no analytical solution and hence iteration method such as Newton method [5], must be used. In order to avoid using an iterative method for solving the non-linear optimization problem, many empirical methods such as McCamy [6] and Qiu [7], look up table (LUT) approach such as Robertson [8] method were proposed for estimating the CCT. For the empirical methods , they are simple, but have poor accuracy. For the LUT approach, its accuracy depends on the number of samples in the LUT. The more points the LUT has, the more accuracy it has. Recently, Ohno [4] proposed a method, which is similar to the Robertson method, uses certain points in CIE $1964(\mathrm{u}, \mathrm{v})$ space on the locus of Blackbody radiator as lookup table (LUT), as shown in Figure 1. The curve (blue) In Figure 1 is the Planckian radiator locus. The lines (solid black) intersecting with the curve are the iso-temperature lines and the intersecting points are all from the LUT. For the given chromaticity (the point $P$ shown in Figure 1) of the light source, the nearest two points from the LUT can be found, suppose they are the points $P_{j}$ and $P_{j+1}$. The two associated isotemperature lines $L_{j}$ and $L_{j+1}$ intersects at point $o$. Note that points on the line $L_{j}$ nearer the Planckian radiator locus has the known CCT of $T_{j}$. Similarly, points on the line $L_{j+1}$ nearer the Planckian radiator locus has the known CCT of $T_{j+1}$. Linking the point $P$ and $o$ forms a line $L$ (dotted line shown in Figure 1 ). The line $L$ and the line $L_{j}$ forms an angle $\theta$. If the line $L$ and the line $L_{j}$ coincides, the angle $\theta$ is zero. Hence in this case, the CCT should be $T_{j}$. If the line $L$ and the line $L_{j+1}$ coincides, the 
associated CCT should be $T_{j+1}$. Robertson method for finding CCT of the given light source uses linear interpolation on angle $\theta$. While for the Ohno method, firstly, a third nearest to the given point $P$ from the LUT can be found. Let it be the point $P_{j+2}$. The distance $D\left(P, P_{j+1}\right)$ (between $P$ and $\left.P_{j+1}\right)$ is the smallest among the three distances: $D\left(P, P_{k}\right), k=j, j+1, j+2 . \mathrm{A}$ line $L_{O}$ going through points $P_{j}$ and $P_{j+2}$ can be established. Let $P_{x}$ be such a point on line $L_{O}$ so that the line segment linking points $P$ and $P_{x}$ and the line $L_{O}$ are perpendicular. A shortest distance to the line $L_{O}$ from the given point $P$ is simply $D\left(P, P_{x}\right)$, which is an estimation to the true $\left|D_{u v}\right|$, denoted by $D_{u v 1}$. The associated CCT, denoted as $T_{u v 1 e}$, can be found using the linear interpolation again depending on the distances between $P_{j}$ and $P_{x}$, and between $P_{j+2}$ and $P_{x}$. Ohno suggested that a scaling factor should be applied to the estimated CCT $T_{u v l e}$, because of the nonlinear property of the Planckian radiator locus. The corrected CCT, denoted as $T_{u v 1}$, is given by

$$
T_{u v 1}=0.99991 T_{u v 1 e}
$$

Note that $D_{u v 1}$ has a sign associated it (see Eq. 4). Ohno gave an estimation $v\left(T_{u v 1}\right)$ (see Eq. 8 in Ohno paper [4]). Hence, the sign (see Eq. 4) can be computed. Ohno [4] derived the formulae based on the (green) triangle shown in Figure 1. Hence, the method is called the Triangle solution.

Next, let $D(P,(u(T), v(T))$ be the distance between $P$ and the point $(u(T), v(T))$ on Planckian radiator locus. $D(P,(u(T), v(T))$ can be estimated locally using a second order polynomial, denoted as $D_{2}(P,(u(T), v(T))$, of the colour temperature $T$, i.e.

$$
D_{2}\left(P,(u(T), v(T))=a_{0}+a_{1} T+a_{2} T^{2}\right.
$$

based on the facts: distances $D_{2}\left(P, P_{k}\right)$ correspond to $T_{k}$ for $k=j, j+1$ and $j+2$. Suppose $D_{2}\left(P,(u(T), v(T))\right.$ defined by Eq. 6 reaches its minimum at $T_{u v 2}$ (which is the estimation of the CCT for the given light source), and $D_{2}\left(P,\left(u\left(T_{u v 2}\right), v\left(T_{u v 2}\right)\right)\right.$ is an estimation to the true $\mid D_{u v}$ for the given light source and it is named as $D_{u v 2}$. Once again $D_{u v 2}$ has a sign associated it (see Eq. 4). Finally,

$$
\begin{aligned}
& D_{u v}=D_{u v 1}, T_{u v}=T_{u v 1} \quad \text { if } \quad\left|D_{u v 1}\right|<0.002 \\
& D_{u v}=D_{u v 2}, T_{u v}=T_{u v 2} \quad \text { otherwise }
\end{aligned}
$$

Note that the estimated $T_{u v 2}$ is also corrected using the same scaling factor given in Eq. 5 as Ohno suggested for the same reason. Note that Ohno [4] called the method using second order interpolation (see Eq. 6) as parabolic solution. Note also that when computing the sign associated with $D_{u v 2}, v\left(T_{u v 2}\right)$ is needed. Ohno [4] suggested either using $v\left(T_{u v 2}\right)=v\left(T_{u v 1}\right)$ or computing $v\left(T_{u v 2}\right)$ using the Eqs. 8 and 9 directly below:

$$
\begin{aligned}
& X(T)=k \int_{\lambda_{0}}^{\lambda_{n}} M(\lambda, T) \bar{x}(\lambda) d \lambda=k \sum_{j=0}^{471} M\left(\lambda_{j}, T\right) \bar{x}\left(\lambda_{j}\right), \\
& Y(T)=k \int_{\lambda_{0}}^{\lambda_{n}} M(\lambda, T) \bar{y}(\lambda) d \lambda=k \sum_{j=0}^{471} M\left(\lambda_{j}, T\right) \bar{y}\left(\lambda_{j}\right), \\
& Z(T)=k \int_{\lambda_{0}}^{\lambda_{n}} M(\lambda, T) \bar{Z}(\lambda) d \lambda=k \sum_{j=0}^{471} M\left(\lambda_{j}, T\right) \bar{Z}\left(\lambda_{j}\right),
\end{aligned}
$$

where $\lambda_{0}=360 \mathrm{~nm}, \lambda_{n}=830 \mathrm{~nm}, \lambda_{j+1}-\lambda_{j}=1 \mathrm{~nm}$, and the function $M$ is given in Eq. $1, \bar{x}(\lambda), \bar{y}(\lambda)$, $\bar{Z}(\lambda)$ are the CIE 1931 colour matching functions. The $k$ in Eq. 7 is a scaling factor. However, 
it is not important since it will be canceled when the chromaticity coordinates $u(T)$ and $v(T)$ are computed.

$$
\begin{aligned}
& u(T)=4 X(T) /[X(T)+15 Y(T)+3 Z(T)], \\
& v(T)=6 Y(T) /[X(T)+15 Y(T)+3 Z(T)] .
\end{aligned}
$$

\section{The Proposed Method}

Though, finding the minimum of the distance $D_{2}(P,(u(T), v(T))$ given by Eq. 6 is not a difficult task, it looks awkward, since we use three points $P_{k}, k=j, j+1$ and $j+2$, for the interpolation to get the $D_{2}\left(P,(u(T), v(T))\right.$ given by Eq. 6 and we might expect the minimum point $T_{u v 2}$ appears in the interval $\left(T_{j}, T_{j+1}\right)$, which is not guaranteed in general. On the other hand, third order interpolation may be better than second order for approximating the true distance $D(P,(u(T), v(T))$. So an additional point, the fourth nearest point from the LUT to the given point $P$, can be selected. Let the point be $P_{j-1}$. Now using the four points $P_{k}$, $k=j-1, j, j+1$ and $j+2$, and the associated colour temperatures $T_{k}$, a third order polynomial $D_{3}(P,(u(T), v(T))$ of the colour temperature $T$ can be formed and it can be written as:

$$
D_{3}\left(P,(u(T), v(T))=a_{0}+a_{1} T+a_{2} T^{2}+a_{3} T^{3}\right.
$$

Thus, $D_{3}(P,(u(T), v(T))$ can be considered as an approximation to the true distance $D\left(P,(u(T), v(T))\right.$. We expect $D_{3}(P,(u(T), v(T))$ is locally closer to the true distance $D\left(P,(u(T), v(T))\right.$ than $D_{2}\left(P,(u(T), v(T))\right.$ does since we have used more points. Let $D_{3}(P,(u(T), v(T))$ reaches its minimum at colour temperature $T_{u v 3}$ and denote $D_{3}\left(P,\left(u\left(T_{u v 3}\right), v\left(T_{u v 3}\right)\right)\right.$ and the associated distance with sign (see Eq. 4) as $D_{u v 3}$. Thus, we expect $D_{u v 3}$ is closer to the true $D_{u v}$ than $D_{u v 2}$ does. This approach is named as third order solution as well.

Figure 2 shows the performance of the triangle (blue curve), parabolic (green cureve) and third order (red curve) solutions with samples selected on the isotemperature with colour temperature $T=1000 \mathrm{~K}$. For each sample selected, each of the method should give a CCT prediction $T_{P}$ and $D_{u v}$ prediction $D_{u v P}$. The absolute differences $|\Delta T|=\left|T-T_{P}\right|$ and $\left|\Delta D_{u v}\right|=\left|D_{u v}-D_{u v P}\right|$ are the measures of the performance for each method. Horizontal axis is the true $\left|D_{u v}\right|$ and vertical axis is the absolute error $|\Delta T|$ for each of the method. The blue, green and red curves are the prediction errors for the triangle, parabolic and third order solutions respectively. It can be seen that when $\left|D_{u v}\right|<0.005$, the triangle solution give the best prediction. When $\left|D_{u v}\right|$ is about between 0.005 and 0.012 , the parabolic solution gives the best prediction. When $\left|D_{u v}\right|$ is about larger than 0.012 , the third order solution is the best. Note that the thin vertical dotted line is $\left|D_{u v}\right| \equiv 0.002$, which is a threshold for Ohno combined method, that is to say, when $\left|D_{u v}\right|$ predicted by the triangle solution is smaller than this threshold, the triangle solution is used, otherwise, the parabolic solution is used. From this Figure, it tells that Ohno (combined) method may make the wrong decision for $\left|D_{u v}\right|$ between 0.002 and 0.005 since it can be better if the triangle solution is used for this range as well. 


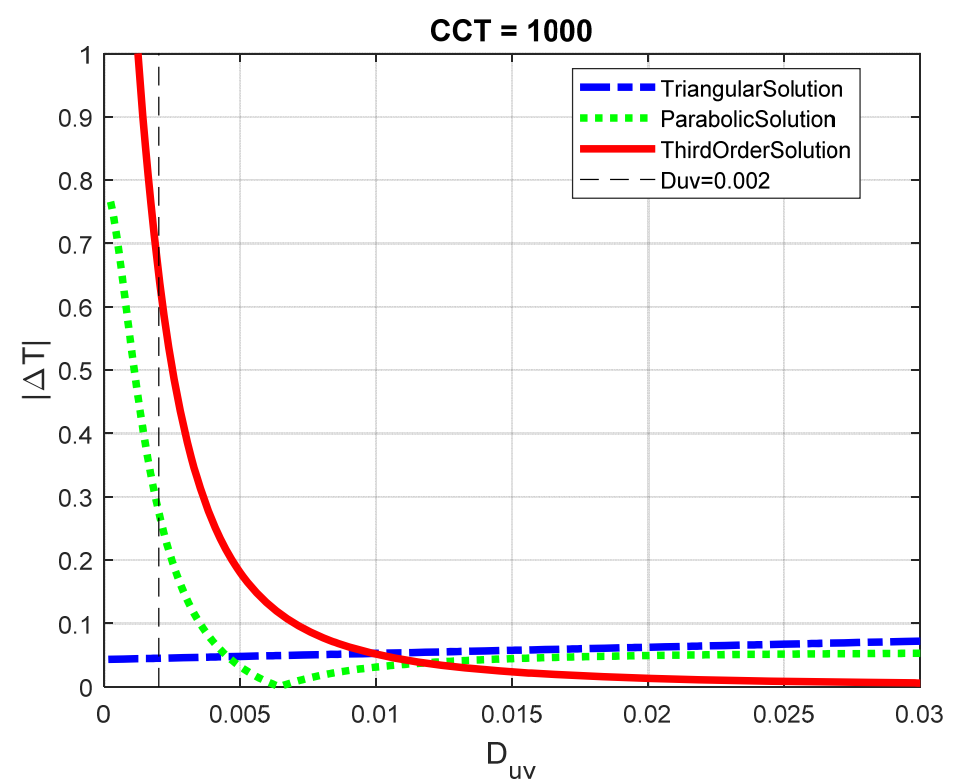

Figure 2 - Performance for each of the Triangle, Parabolic and Third Order solutions in predicting CCT

Motivated by Figure 1, we propose a hybrid method, which is defined by the following:

$$
\begin{array}{ll}
D_{u v}=D_{u v 1}, T_{u v}=T_{u v 1} & \text { if }\left|D_{u v 1}\right|=\min \left(\left|D_{u v 1}\right|,\left|D_{u v 2}\right|,\left|D_{u v 3}\right|\right) \\
D_{u v}=D_{u v 2}, T_{u v}=T_{u v 2} & \text { if }\left|D_{u v 2}\right|=\min \left(\left|D_{u v 1}\right|,\left|D_{u v 2}\right|,\left|D_{u v 3}\right|\right) \\
D_{u v}=D_{u v 3}, T_{u v}=T_{u v 3} & \text { if }\left|D_{u v 3}\right|=\min \left(\left|D_{u v 1}\right|,\left|D_{u v 2}\right|,\left|D_{u v 3}\right|\right)
\end{array}
$$

Note in the proposed method for each of the parabolic and third order solutions, an associated CCT $T_{u v i}$ can be estimated first, then the $D_{u v i}$ is computed using the given chromaticity point $P$ and the point $\left(u\left(T_{u v i}\right), v\left(T_{u v i}\right)\right)$ on the Planckian radiator locus. While for the triangle solution, $D_{u v 1}$ is the predicted distance using formula (see Eq. 8 in [4]) given by Ohno. In the next section, the proposed method will be tested and compared with the Ohno combined method.

\section{Performance of the proposed method}

We tested the performance of the proposed method together with the Ohno method by using sets of samples (hypothetical light sources) with known CCTs and $D_{u v}$. Specifically, we considered sets of 5 samples placed on different isotemperature lines, which by definition must have the same CCT for a perfect computational method. For any selected colour temperature $T$ from $1000 \mathrm{~K}$ to $20000 \mathrm{~K}$ at $1 \mathrm{~K}$ step, the points $(u(T), v(T))$ on the Planckian radiator locus were computed, and the associated isotemperature lines were determined.

Then, we computed the five equi-spaced samples in each isotemperature line: sample \#3 was placed exactly on the Planckian radiator locus in the $u, v$ space; samples \#1 and \#2 were placed above the Planckian locus at distances of $3 \times 10^{-2}$ and $1.5 \times 10^{-2}$ units in $u, v$ space, respectively; samples \#4 and \#5 were placed below the Planckian locus at distances of $1.5 \times 10^{-2}$ and $3 \times 10^{-2}$ units in $u, v$ space, respectively. The distance of $3 \times 10^{-2}$ units to the Planckian locus in the $u, v$ space was used by Ohno [4] and for comparison purpose it is used again in this paper.

The maximum absolute difference between the predicted and exact CCTs of the five aforementioned samples on the isotemperature line will be designated as $\max |\Delta T|$ and it can be considered to be the maximum prediction error for the isotemperature line. 
Figure 3 shows the maximum prediction errors $(\max |\Delta T|)$ for the Ohno (blue) and proposed (red) methods. It can be seen that in general, the maximum prediction error is increasing with the colour temperature. When the colour temperature is close to $20000 \mathrm{~K}$, the maximum prediction error is about 1.2 for the Ohno method and 0.4 for the proposed method. Details can be observed by enlarge parts of the Figure. Figures 4 shows parts of Figure 3 around $1020 \mathrm{~K}$ (left diagram), $8780 \mathrm{~K}$ (middle diagram) and $15950 \mathrm{~K}$ (right diagram) respectively. From each of them it can be seen that the blue curve is above or overlapping with the red curve, which means the proposed method outperforms the Ohno method.

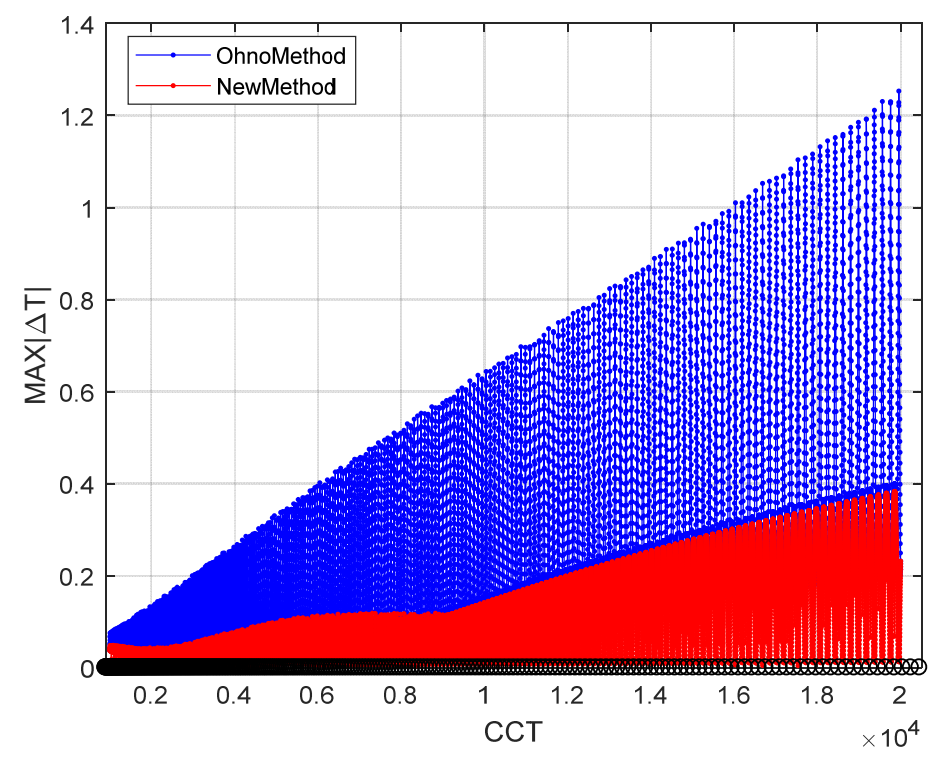

Figure 3 - Performance for Ohno (blue) and proposed (red) methods in predicting CCT

For the absolute difference between the predicted and exact $D_{u v}$, it was found that predictions from both methods are good. With the samples \#1 and \#5, on each isotemperature lines have exact $D_{u v}= \pm 0.03$, both methods have $\max \left|\Delta D_{u v}\right|<4 \cdot 10^{-7}$ as shown in the left diagram in Figure 5. With the samples \#2 and \#4, on each isotemperature lines have exact $D_{u v}= \pm 0.015$, again both methods have $\max \left|\Delta D_{u v}\right|<10^{-6}$ as shown in the middle diagram in Figure 5. It can be seen in both cases, the proposed method is better than the Ohno method. With the samples \#3 on each isotemperature lines have exact $D_{u v}=0$, both methods have the $\max \left|\Delta D_{u v}\right|<10^{-5}$ as shown in the right diagram in Figure 5 . It can be seen in this case, the proposed method is better than the Ohno method in most cases. Overall, the proposed method has also a better prediction accuracy than the Ohno method does in predicting $D_{u v}$.

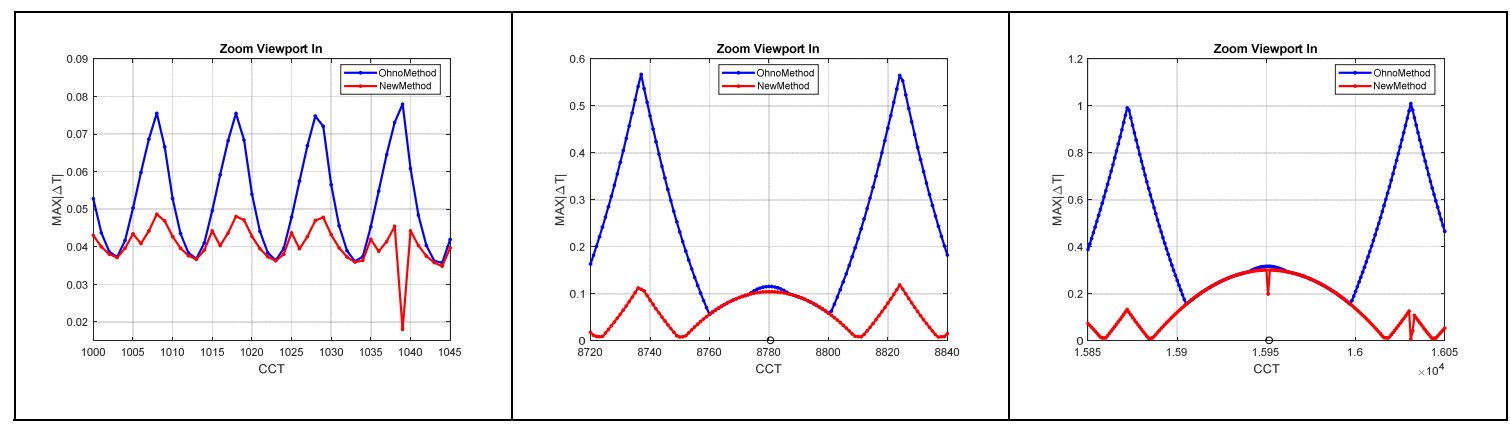

Figure 4 - Enlarge parts of Figure 3 around 1020K(left diagram), 8780K (middle diagram)and $15950 \mathrm{~K}$ (right diagram) respectively 


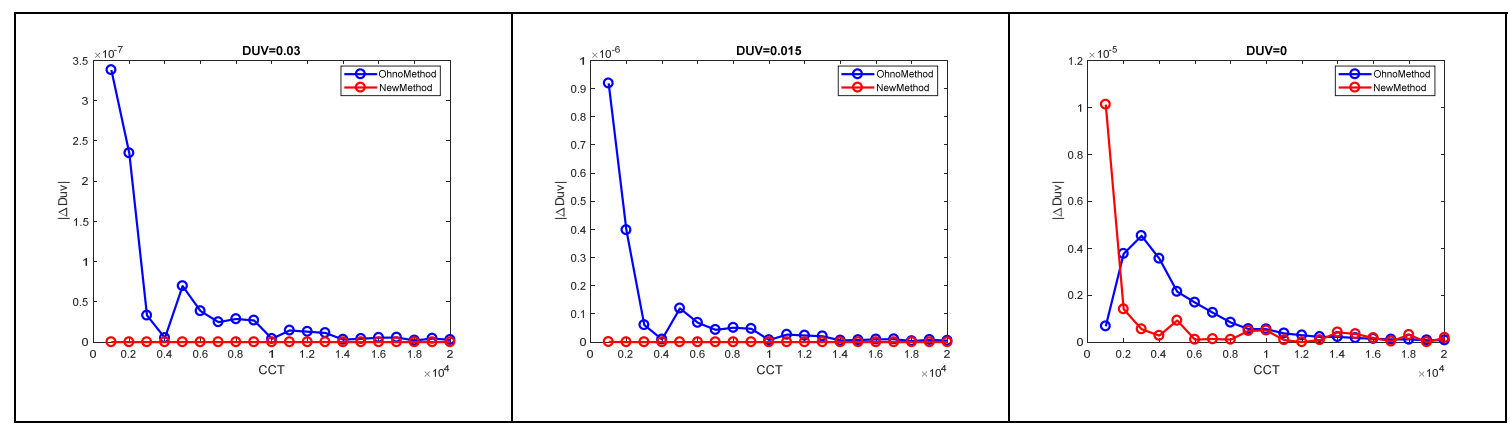

Figure 5 - Performance for Ohno (blue) and proposed (red) method in predicting $D_{u v}$

\section{Conclusions}

In this paper, a new method is proposed for computing CCT and Duv for a given light source. The proposed method consists of the triangle, parabolic and third order solutions. The method is simple and is a generalization of the Ohno method. Testing results show the proposed method is more accurate than the Ohno method in predicting the CCT and $D_{u v}$.

\section{Acknowledgment}

The work is supported by National Natural Science Foundation of China (Grant number: 61575090, 61775169)

\section{References}

[1] Wyszecki, G. W. and Stiles, W. S. 1967. Colour Science: Concepts and Methods, Quantitative data and formulas. New York: Wiley.

[2] CIE 1931. Proceedings of the 8th Session, International Commission on Illumination. pp. 19-29, Cambridge.

[3] CIE 2018. CIE 15: 2018. Colourimetry. Paris: CIE.

[4] Ohno, Y. 2014. Practical Use and Calculation of CCT and Duv. LEUKOS. 10(1). 47-55. DOI: $10.1080 / 15502724.2014 .839020$.

[5] Changjun Li, Guihua Cui, Manuel Melgosa, Xiukai Ruan, Yaoju Zhang, Long Ma, Kaida Xiao, and M. Ronnier Luo. 2016. Accurate method for computing correlated colour temperature, Optics Express. 24(13).14066-14078.

[6] McCamy, C. S. 1992. Correlated colour temperature as an explicit function of chromaticity coordinates. Colour Research \& Application. 17(2), 142-144.

[7] Qiu, X. 2010. Formulas for computing correlated colour temperature. Colour Research \& Application. 12(5). 285-287.

[8] Robertson, A. R. 1968. Computation of the correlated colour temperature and distribution temperature. Journal of the Optical Society of America. 58(11). 1528-1535. 